\title{
HYDROCHEMICAL CONDITIONS OF THE ŁOSOSINA RIVER WATER MANAGEMENT IN THE AREA OF TYMBARK
}

\author{
Agnieszka Policht-Latawiec ${ }^{1}$, Włodzimierz Kanownik ${ }^{1}$ \\ 1 Department of Land Reclamation and Environmental Development, University of Agriculture in Krakow, \\ Mickiewicza Av. 24-28, 30-059 Krakow, Poland, e-mail: a.policht@ur.krakow.pl; rmkanown@cyf-kr.edu.pl
}

Received: 2015.09 .05

Accepted: 2015.10.06

Published: 2015.11.10

\begin{abstract}
Sustainable use of waters requires not only determining the amount, but primarily the quality of the available water resources and developing a long-term programme of their protection. The analysis of the Lososina river water in the area of Tymbark city was presented in the paper. The water was tested in a view of the requirements as the natural fish habitat and its potential use for people supply in potable water. The river water samples were taken in 2014 at randomly selected dates, once a month in 5 measurement points. 21 physicochemical indices were assessed in the samples. The assessment of the Lososina river water quality was made on the basis of the results of both: on site and laboratory testing, which were compared with the Regulation of the Minister of Environment of 23 October 2014. The utility values were assessed on the basis of the Regulations of the Minister of Environment of 27 November and 04 October 2014. The analysis of the results demonstrated that the Łososina river water met the requirements of quality class I water in points 1, 2 and 3. Below Tymbark the Łososina river water was polluted, so due to high $\mathrm{BOD}_{5}$ in points 4 and 5, and phosphate concentrations in point 4 , it was classified as class II, i.e. good state. Pollution coefficients computed according to Burchard and Dubaniewicz classify the Łososina river water as clean along the whole investigated stretch. Below Tymbark city (points 4 and 5) the Łososina river water cannot be used for drinking water supply because of high $\mathrm{BOD}_{5}$ and iron concentrations. In the other points it could be used for water supply following appropriate physical and chemical treatment. The water does not meet the requirements for salmonid or cyprinid fish along the whole stretch because of high nitrite concentrations, except point 3, where the Lososina river water provided a proper natural habitat for carp.
\end{abstract}

Keywords: water quality, physicochemical indices, water management, the Łososina river, Tymbark city.

\section{INTRODUCTION}

Water management plays a crucial role in stimulating sustainable development of rural areas [Bourne 2002, GLP 2005, EEA 2010, Ahiablame et al. 2012, Mladenović-Ranisavljevića et al. 2012, Radu et al. 2013]. It is also a major element of the natural environment protection [Zampella and Procopio 2009, Erle and Robert 2010, Zieliński et al. 2012, Chomutowska and Wilamowski 2014]. Proper quality water available to the community may considerably affect the development of tourist and leisure activities [Grochowska and Tan- dyrak 2007], agricultural and food industries, fish farming, organic farming or construction of hydro power plants [Bodgał et al. 2012]. The intended water use poses requirements for its appropriate characteristics [Policht-Latawiec et al. 2014]. The highest requirements characterise potable water, whereas waters destined for other purposes (agriculture, industry or leisure activities) should also meet appropriate criteria, such as values characterizing chemical, physical and biological water properties [Kruk 2007, Czaban 2008, Kanownik et al. 2011, Bogdał et al. 2015]. Human activity, climatic conditions and natural components, such as 
the substratum or vegetal cover, considerably influence the development of water properties in the catchment [Derwich et al. 2010, Raczyńska et al. 2013, Kanownik and Policht-Latawiec 2015]. Deterioration of water quality in the catchment may be caused by pollutants from industrial and municipal sewage, chemicals used in agriculture and forestry or transport pollution [Schoonover et al. 2006, Kiryluk and Rauba 2011, Policht-Latawiec and Kanownik 2013]. A number of collective activities, aimed at ensuring rational management of surface waters leads to protection of water resources [Ostrowski 2010, Albrecht 2013]. An important objective is preventing the disturbance of natural balance and any changes which would cause water uselessness for people, animals or vegetation [Zhang et al. 2013, Policht-Latawiec et al. 2015]. According to hydrochemical research, smaller rivers (excluding the rivers flowing through the industrial and urbanised areas) have waters of significantly better quality than bigger rivers [El-Guamri and Belghyti 2006, Sojka and Murat-Błażejewska 2009]. Unfortunately, owing to untreated sewage discharges from farms and rural areas and area pollution due to farming, a vast portion of these rivers is considerably polluted [Kuźniar et al. 2009, Policht-Latawiec 2012, Radu et al. 2014].

\section{MATERIAL AND METHODS}

The hydrochemical research of the Łososina river was conducted in 2013. The river is 56.915 $\mathrm{km}$ long with average bottom slope $9.5 \%$.
The Łososina springs are located in the Beskid Wyspowy Mts., at the altitude of about $960 \mathrm{~m}$ a.s.l. It flows from the north-eastern slopes of Jasien in Półrzeczki village and flows into Czchów Lake in Witowice Dolne village. The lake was formed as a result of the Dunajec river damming on the dam in Czchów. The watercourse is flowing in the bed composed of pebbles, gravels, sands and sandy alluvions [Paczyński and Sadurski 2007]. According to the surface waters typology, the Lososina to the Słopniczanka river is a flysch stream, whereas from the Słopniczanka to its mouth is a small flysch river. The river catchment, of $410.60 \mathrm{~km}^{2}$ is situated at the altitude of 233-1052 m a.s.l. in the Malpolskie voivodship. According to Kondracki's [2013] geographical division, almost whole catchment is situated in the Beskid Wyspowy Mts, only a small northeastern part belongs to the Wiśnicz Upland.

Water for analyses was sampled on 11 dates from $3845 \mathrm{~m}$ of the Lososina river stretch in the Tymbark city [ISO 5667-6], in 5 measurement points (Figure 1): above the city at $\mathrm{km} 41+022$ (point 1) and at $\mathrm{km} 40+272$ (point 2), in the city area at $39+322$ (point 3 ) and below the city at $\mathrm{km}$

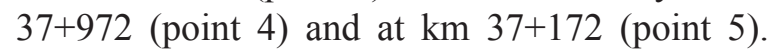
Both the upper and the lower part of the investigated catchment are used as farmlands and forests, whereas the central part are urban and settlement areas posing a potential pollution hazard.

Water $\mathrm{pH}$ was assessed on site using CP-104 $\mathrm{pH}$ meter, electrolytic conductivity by means of CC-102 conductometer, dissolved oxygen concentration and the degree of water saturation

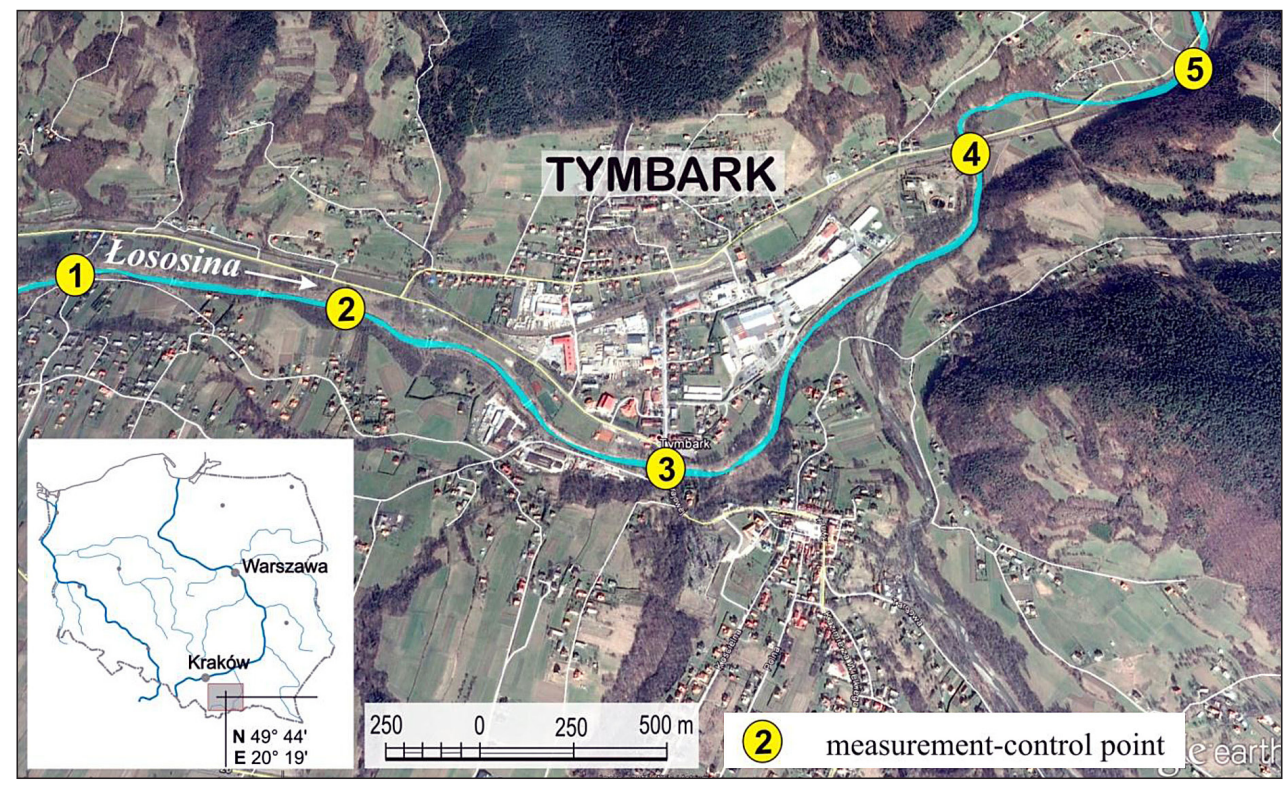

Figure 1. Location of measurement and control points on the investigated section of the Łososina river 
with oxygen - using CO-411 oxygen meter, and total dissolved solids using TDS meter $(\mathrm{HACH}$ LANGE).

In the laboratory, total suspended solids were determined by drying and weighing method and concentrations of $\mathrm{Ca}^{2+}, \mathrm{Mg}^{2+}, \mathrm{Na}^{+}, \mathrm{K}^{+}, \mathrm{Fe}$ and $\mathrm{Mn}$ ions by means of atomic absorption spectrometry (ASA) on UNICAM SOLAR 969 spectrometer. Biochemical oxygen demand $\left(\mathrm{BOD}_{5}\right)$ was determined by Winkler's method and chemical oxygen demand (COD-Mn) by titration in $\mathrm{KMnO}_{4}$. Concentrations of ammonium nitrogen $\left(\mathrm{N}_{-} \mathrm{NH}_{4}^{+}\right)$, nitrite $\left(\mathrm{N}-\mathrm{NO}_{2}^{-}\right)$and nitrate $\left(\mathrm{N}-\mathrm{NO}_{3}^{-}\right)$ nitrogen, phosphates $\left(\mathrm{PO}_{4}^{3-}\right)$ and chlorides $\left(\mathrm{Cl}^{-}\right)$ were determined by flow colorimetry analysis on FIAstar 5000 apparatus, sulphates $\left(\mathrm{SO}_{4}^{2-}\right)$ by precipitation method [Rozporządzenie MŚ 2013]. Concentrations of $\mathrm{NH}_{4}^{+}, \mathrm{NO}_{2}^{-}$and $\mathrm{NO}_{3}^{-}$were computed from the nitrogen forms determined in the laboratory.

While elaborating the results, the minimum and maximum values for individual indices were determined and arithmetic mean was computed. Water quality was assessed in compliance with The Regulation of the Minister of Environment of 22 October 2014 establishing the way of classifying the state of uniform parts of surface waters and environmental quality standards for priority substances [Rozporządzenie MŚ 2014], whereas the utility value through comparing the results of assessments with the values permissible for water intended for supply to people [Rozporządzenie MŚ 2002b] and for natural fish habitats [Rozporządzenie MŚ 2002a].

Water pollution was also assessed using Burchard and Dubaniewicz formula [1981]:

$$
W Z=\frac{\sum_{1}^{2} \frac{S D_{t}}{S W_{t}}+\sum_{1}^{n-2} \frac{S W_{n-2}}{S D_{n-2}}}{n}
$$

where: $W_{z}$-pollution coefficient,

$S D_{t}^{z}-$ concentration of dissolved oxygen permissible for class I quality waters, in compliance with regulation of 2014 ,

$S W_{t}$ - dissolved oxygen concentration, average for the research period,

$S W_{n-2}$ - values of the other indices considered, average for the research period, $S D_{n-2}$ - values of the other indices considered, admissible for class I quality waters according to the regulation of 2014 , $n$ - number of the indices considered in the calculation.
According to the pragmatics of the method, water is considered clean if the pollution coefficient $\mathrm{W}_{\mathrm{z}}$ is lower than 0.75 . For the coefficient values $0.76-1.00 ; 1.01-1.50 ; 1.51-2.00$ and over 2 , the water is regarded, respectively as: slightly polluted, clearly polluted, strongly polluted and sewage. The level of linear dependence between the electrolytic conductivity and concentrations of some water quality indices in the Łososina river was determined in the paper. The dependence was stated as Pearsons's linear correlation coefficient in the closed interval [-1,1] [Buda and Jarynowski 2010].

\section{RESULTS}

The Lososina river water temperature during the investigated period ranged from 1.9 to $16.3{ }^{\circ} \mathrm{C}$ and its $\mathrm{pH}$ from acid ( $\mathrm{pH}$ 6.2) to alkaline (pH 8.5). Total dissolved solids content in point 2 sporadically exceeded the value permissible for class I and its average value $\left(8.0 \mathrm{mg} \cdot \mathrm{dm}^{-3}\right)$ was the highest in comparison with other analysed points. Yet, it remained on the level of quality class I [Rozporządzenie MŚ 2014]. Over the whole investigated period the river water revealed very good oxygen conditions - average degree of oxygen saturation was over $94 \%$ and did not exceed $103 \%$, while average concentration of dissolved oxygen was over $9.3 \mathrm{mg} \cdot \mathrm{dm}^{-3}$. Only five-day biochemical oxygen demand $\left(\mathrm{BOD}_{5}\right)$ in points 4 and 5 (below Tymbark city) exceeded the limit value for class I $-3.0 \mathrm{mg} \cdot \mathrm{dm}^{-3}$. On the other hand, COD-Mn was below the limit value for class I along the whole analysed length of the Lososina river. Electrolytic conductivity of water was the highest in points 4 and 5 - it did not exceed $448 \mu \mathrm{S} \cdot \mathrm{cm}^{-1}$, whereas the maximum dissolved solids concentration was $340 \mathrm{mg} \cdot \mathrm{dm}^{-3}$ (Table 1). These values were lower than the limit values for quality class I waters, which evidences a low mineral pollution of water. The Łososina river water salinity was on a low level; the highest concentration of sulphates $\left(\mathrm{SO}_{4}^{2-}\right)$ was 32 $\mathrm{mg} \cdot \mathrm{dm}^{-3}$, chlorides $\left(\mathrm{Cl}^{-}\right)-27 \mathrm{mg} \cdot \mathrm{dm}^{-3}$, calcium $\left(\mathrm{Ca}^{2+}\right)-85 \mathrm{mg} \cdot \mathrm{dm}^{-3}$, magnesium $\left(\mathrm{Mg}^{2+}\right)-12.2$ $\mathrm{mg} \cdot \mathrm{dm}^{-3}$, sodium $\left(\mathrm{Na}^{+}\right)-47.5 \mathrm{mg} \cdot \mathrm{dm}^{-3}$ and potassium $\left(\mathrm{K}^{+}\right)-5.5 \mathrm{mg} \cdot \mathrm{dm}^{-3}$. Average and maximum concentrations of ammonium nitrogen did not exceed the limit value for quality class I $\left(0.78 \mathrm{mg} \cdot \mathrm{dm}^{-3}\right)$. In case of phosphates concentration, the average values did not exceed the 
Table 1. Range and average values of physicochemical indices and water quality class in the Lososina river

\begin{tabular}{|c|c|c|c|c|c|c|c|c|c|c|c|c|}
\hline \multirow{3}{*}{ Indices } & \multicolumn{5}{|c|}{ Range } & \multirow{2}{*}{\multicolumn{5}{|c|}{ Average }} & \multirow{2}{*}{\multicolumn{2}{|c|}{$\begin{array}{c}\text { Limit values } \\
\text { for class } \\
\text { [Rozporządzeni } \\
\text { MŚ 2014] }\end{array}$}} \\
\hline & \multicolumn{5}{|c|}{ Measurement-control point } & & & & & & & \\
\hline & 1 & 2 & 3 & 4 & 5 & 1 & 2 & 3 & 4 & 5 & I & II \\
\hline \multicolumn{13}{|c|}{ Physical indices } \\
\hline Temperature $\left[{ }^{\circ} \mathrm{C}\right]$ & $2.3-15.4$ & $1.9-15.5$ & $2.0-15.9$ & $2.4-16.3$ & $2.5-15.6$ & 12.1 & 11.8 & 12.0 & 12.5 & 12.3 & $\leq 22$ & $\leq 24$ \\
\hline $\begin{array}{l}\text { Total suspended solids } \\
{\left[\mathrm{mg} \cdot \mathrm{dm}^{-3}\right]}\end{array}$ & $1.8-22.5$ & $1.1-28$ & $1.2-12.5$ & $1.0-13.9$ & $1.2-13.0$ & 7.7 & 8.0 & 5.1 & 4.5 & 4.4 & $\leq 25$ & $\leq 50$ \\
\hline \multicolumn{13}{|c|}{ Oxygen-related indices } \\
\hline $\begin{array}{l}\text { Dissolved oxygen [mg } \\
\left.\mathrm{O}_{2} \cdot \mathrm{dm}^{-3}\right]\end{array}$ & $8.4-10.6$ & $8.6-10.4$ & $8.2-10.5$ & $7.2-10.7$ & $7.4-12.5$ & 10.2 & 9.7 & 9.3 & 9.4 & 9.8 & $\leq 7$ & $\leq 5$ \\
\hline $\begin{array}{l}\text { Oxygen saturation degree } \\
{[\%]}\end{array}$ & $92-108$ & $90-122$ & $71-112$ & $81-122$ & $83-144$ & 102 & 103 & 94 & 98 & 103 & - & \\
\hline $\mathrm{BOD}_{5}\left[\mathrm{mg} \mathrm{O}_{2} \cdot \mathrm{dm}^{-3}\right]$ & $0.3-2.9$ & $0.9-3.9$ & $0.6-2.5$ & $1.0-10.5$ & $0.7-6.7$ & 1.4 & 1.8 & 1.6 & 3.8 & 3.2 & $\leq 3$ & $\leq 6$ \\
\hline COD-Mn $\left[\mathrm{mg} \mathrm{O}_{2} \cdot \mathrm{dm}^{-3}\right]$ & $3.3-7.1$ & $3.1-6.6$ & $3.5-6.1$ & $4.3-8.6$ & 3.3-7.9 & 5.3 & 4.7 & 4.6 & 5.8 & 5.3 & $\leq 6$ & $\leq 12$ \\
\hline \multicolumn{13}{|c|}{ Salinity } \\
\hline $\begin{array}{l}\text { Electrolytic conductivity } \\
{\left[\mu \mathrm{S} \cdot \mathrm{cm}^{-1}\right]}\end{array}$ & 202-288 & $173-277$ & $177-352$ & $209-448$ & $207-414$ & 240 & 235 & 253 & 294 & 293 & $\leq 1000$ & $\leq 1500$ \\
\hline 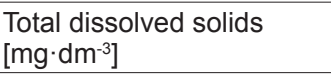 & $157-216$ & $148-219$ & $156-263$ & $176-340$ & $156-315$ & 183 & 174 & 192 & 233 & 224 & $\leq 500$ & $\leq 800$ \\
\hline $\mathrm{SO}_{4}{ }^{2-}\left[\mathrm{mg} \cdot \mathrm{dm}^{-3}\right]$ & $16-27$ & $13-32$ & $16-29$ & $14-25$ & $13-27$ & 21 & 20 & 20 & 19 & 19 & $\leq 150$ & $\leq 250$ \\
\hline $\mathrm{Cl}^{-}\left[\mathrm{mg} \cdot \mathrm{dm}^{-3}\right]$ & $11-16$ & $11-14$ & $12-21$ & $13-27$ & $12-25$ & 14 & 13 & 16 & 17 & 16 & $\leq 200$ & $\leq 300$ \\
\hline $\mathrm{Ca}^{2+}\left[\mathrm{mg} \cdot \mathrm{dm}^{-3}\right]$ & $29-42$ & $24-68$ & $24-85$ & $28-59$ & $27-43$ & 33 & 34 & 38 & 39 & 32 & $\leq 100$ & $\leq 200$ \\
\hline $\mathrm{Mg}^{2+}\left[\mathrm{mg} \cdot \mathrm{dm}^{-3}\right]$ & $5.6-10.2$ & $4.8-10.0$ & $4.9-11.4$ & $6.0-12.2$ & $6.0-12.1$ & 7.4 & 7.1 & 7.6 & 8.4 & 8.3 & $\leq 50$ & $\leq 100$ \\
\hline $\mathrm{Na}^{+}\left[\mathrm{mg} \cdot \mathrm{dm}^{-3}\right]$ & $6.0-10.0$ & $6.8-9.7$ & $6.9-30.1$ & 9.2-47.5 & $8.4-41.3$ & 8.4 & 8.4 & 11.3 & 20.8 & 18.5 & - & - \\
\hline $\mathrm{K}^{+}\left[\mathrm{mg} \cdot \mathrm{dm}^{-3}\right]$ & $2.9-4.0$ & $2.7-3.7$ & $2.9-4.6$ & $3.0-5.5$ & $3.0-5.2$ & 3.5 & 3.1 & 3.5 & 4.2 & 3.9 & - & - \\
\hline \multicolumn{13}{|c|}{ Acidification } \\
\hline Reaction (pH) & $7.4-8.4$ & $7.4-8.5$ & $6.2-8.3$ & $7.4-8.4$ & $6.6-8.5$ & 7.7 & 7.8 & 7.5 & 7.6 & 7.6 & $6-8.5$ & $6-9$ \\
\hline \multicolumn{13}{|c|}{ Biogenic } \\
\hline $\mathrm{PO}_{4}^{3-}\left[\mathrm{mg} \cdot \mathrm{dm}^{-3}\right]$ & $\begin{array}{l}0.03- \\
0.17\end{array}$ & $0.01-0.28$ & $\begin{array}{l}0.02- \\
0.23\end{array}$ & $\begin{array}{l}0.01- \\
0.63 \\
\end{array}$ & $\begin{array}{l}0.00- \\
0.17\end{array}$ & 0.08 & 0.10 & 0.09 & 0.25 & 0.10 & $\leq 0.2$ & $\leq 0.31$ \\
\hline $\mathrm{N}-\mathrm{NH}_{4}{ }^{+}\left[\mathrm{mg} \cdot \mathrm{dm}^{-3}\right]$ & $\begin{array}{c}0.00- \\
0.21\end{array}$ & $0.00-0.70$ & $\begin{array}{l}0.00- \\
0.66\end{array}$ & $\begin{array}{c}0.00- \\
1.18\end{array}$ & $\begin{array}{l}0.00- \\
0.87\end{array}$ & 0.07 & 0.14 & 0.25 & 0.37 & 0.29 & $\leq 0.78$ & $\leq 1.56$ \\
\hline $\mathrm{N}-\mathrm{NO}_{2}^{-}\left[\mathrm{mg} \cdot \mathrm{dm}^{-3}\right]$ & $\begin{array}{l}0.00- \\
0.03\end{array}$ & $0.00-0.03$ & $\begin{array}{l}0.00- \\
0.02\end{array}$ & $\begin{array}{l}0.01- \\
0.05\end{array}$ & $\begin{array}{l}0.01- \\
0.03\end{array}$ & 0.01 & 0.01 & 0.01 & 0.03 & 0.02 & - & \\
\hline $\mathrm{N}-\mathrm{NO}_{3}^{-}\left[\mathrm{mg} \cdot \mathrm{dm}^{-3}\right]$ & $0.1-1.8$ & $0.2-1.5$ & $0.1-1.5$ & $0.4-1.8$ & $0.4-1.5$ & 0.7 & 0.8 & 0.9 & 1.2 & 1.1 & $\leq 2.2$ & $\leq 5$ \\
\hline \multicolumn{13}{|c|}{ Metals } \\
\hline $\mathrm{Fe}\left[\mathrm{mg} \cdot \mathrm{dm}^{-3}\right]$ & $\begin{array}{l}0.11- \\
1.50\end{array}$ & $0.07-1.21$ & $0.03-1.69$ & $\begin{array}{l}0.10- \\
3.35\end{array}$ & $\begin{array}{l}0.06- \\
1.86\end{array}$ & 0.50 & 0.47 & 0.50 & 1.00 & 0.39 & - & \\
\hline $\mathrm{Mn}\left[\mathrm{mg} \cdot \mathrm{dm}^{-3}\right]$ & $\begin{array}{l}0.01- \\
0.42\end{array}$ & $0.00-0.59$ & $0.04-0.39$ & $\begin{array}{l}0.03- \\
0.64 \\
\end{array}$ & $\begin{array}{l}0.02- \\
0.11 \\
\end{array}$ & 0.18 & 0.19 & 0.15 & 0.23 & 0.06 & - & \\
\hline
\end{tabular}

standard for class I $\left(0.2 \mathrm{mg} \cdot \mathrm{dm}^{-3}\right)$ in four investigated points, while in point 4 the value was 0.25 $\mathrm{mg} \cdot \mathrm{dm}^{-3}$. Throughout the period of research, the river water revealed low concentrations of nitrate nitrogen. The average concentration in all measurement points did not exceed the limit value for class I quality waters [Rozporządzenie MŚ 2014]. The highest concentration of total iron (3.35 $\left.\mathrm{mg} \cdot \mathrm{dm}^{-3}\right)$ was registered in point 4 , whereas the lowest $\left(0.03 \mathrm{mg} \cdot \mathrm{dm}^{-3}\right)$ in point 3 . the average $\mathrm{Fe}$ concentration ranged from $0.39-1.00 \mathrm{mg} \cdot \mathrm{dm}^{-3}$.
Manganese $(\mathrm{Mn})$ concentrations fluctuated from 0.01 to $0.64 \mathrm{mg} \cdot \mathrm{dm}^{-3}$ and its average value -0.23 $\mathrm{mg} \cdot \mathrm{dm}^{-3}$ was the highest in point 4 . (Table 1 ).

The assessment of the river water usefulness for potable water supply revealed that among the 21 analysed indices, only 14 were included in the Regulation of the Minister of Environment [2002b]. In all measurement points a majority of the tested indices (the temperature, degree of oxygen saturation of water, COD-Mn, electrolytic conductivity, sulphates, chlorides and nitrates) 
classified the water to A1 category, i.e. water requiring a simple physical treatment (Table 2). As much as $86 \%$ of the samples did not meet the category A requirements, due to high concentration of total suspended solids and ammonium ion $\left(\mathrm{NH}_{4}^{+}\right)$ in point $2, \mathrm{pH}$ in point 3 , phosphates in point 4 , and iron in point 5 . In all the points manganese concentrations caused that the waters were classified to A3 category. The Łososina river water in this point would require a highly efficient physical and chemical treatment. Because of high values of $\mathrm{BOD}_{5}$ and $\mathrm{Fe}, 10.5$ and $3.35 \mathrm{mg} \cdot \mathrm{dm}^{-3}$ respectively, only the water immediately below the city (point 4) could not be taken for potable water supply. High concentrations of manganese and iron are typical for the surface waters of the Malopolskie voivodship. Their origin is natural, associated with the catchment geological structure, chemical composition of rocks and soils in the catchment and riverbeds [Kanownik et al. 2013].

Waters of the Lososina river were assessed in the paper as natural habitat for the salmonid and cyprinid fish. On the basis of 7 physicochemical indices it was found that water in all points did not meet the requirements for the salmonids because of high nitrite concentrations. On the other hand, water fulfilled the requirements for cyprinid fish only in point 3 [Rozporządzenia MŚ 2014]. Below the city (points 4 and 5) the Łososina river water did not meet the requirements for fish, not only due to high nitrite concentrations but also because of $\mathrm{BOD}_{5}$ values and ammonium nitrogen concentration. The other indices: water temperature, total suspended solids, dissolved oxygen and $\mathrm{pH}$ met the standards for the natural habitat for salmonid and cyprinid fish.

Surface water susceptibility to pollution may be determined using pollution coefficient $\mathrm{W}_{\mathrm{z}}$, which classifies waters to a given category. The analysis of 14 indices showed that the Lososina river water was clean, since the value of the pollution coefficient along the analysed river stretch did not exceed 0.75 (Table 3). A higher value of the coefficient in points 4 and 5 may be explained by a local pollution connected with these points location below the city of Tymbark. It is apparent particularly for oxygen ( $\mathrm{BOD}_{5}$ and COD-Mn) and biogenic $\left(\mathrm{PO}_{4}^{3-}\right)$ indices.

Correlations between electrolytic conductivity and individual water quality indices concentrations may be interpreted as strongly positive for TDS, $\mathrm{Mg}^{2+}, \mathrm{Na}^{+}, \mathrm{K}^{+}$, strongly negative (TSS, $\mathrm{Fe}$ ), weakly positive $\left(\mathrm{Cl}^{-}\right)$and weakly negative for $\left(\mathrm{SO}_{4}^{2-}, \mathrm{Ca}^{2+}\right.$ and $\left.\mathrm{Mn}^{2+}\right)$ indices. Statistically

Table 2. Frequency of occurrence [\%] of indices used for assessment of water usefulness for potable water supply for people

\begin{tabular}{|c|c|c|c|c|c|c|c|c|c|c|c|c|c|c|c|c|c|}
\hline \multirow{4}{*}{ Indices } & \multirow{3}{*}{\multicolumn{3}{|c|}{$\begin{array}{c}\text { Admissible values of } \\
\text { indexes for individual } \\
\text { water categories } \\
\text { [Rozporządzenie MŚ } \\
\text { 2002b] }\end{array}$}} & \multicolumn{14}{|c|}{$\begin{array}{l}\text { Frequency of index values (\% of samples) in normative range } \\
\text { for a given water treatment category }\end{array}$} \\
\hline & & & & \multicolumn{14}{|c|}{ Measurement-control point and quality categories } \\
\hline & & & & \multicolumn{3}{|c|}{1} & \multicolumn{2}{|r|}{2} & \multicolumn{3}{|c|}{3} & \multicolumn{3}{|c|}{4} & \multicolumn{3}{|c|}{5} \\
\hline & $\mathrm{A} 1$ & A2 & A3 & A1 & \begin{tabular}{l|l} 
A2 & A
\end{tabular} & A3 A & A1 $A$ & \begin{tabular}{l|l} 
A2 & A3
\end{tabular} & A1 & A2 & A3 & A1 & $\mathrm{A} 2$ & A3 & A1 & A2 & A3 \\
\hline Temperature $\left[{ }^{\circ} \mathrm{C}\right]$ & $25^{1}$ & $25^{1}$ & $25^{1}$ & \multicolumn{2}{|r|}{100} & & \multicolumn{2}{|c|}{100} & \multicolumn{3}{|c|}{100} & \multicolumn{3}{|c|}{100} & \multicolumn{3}{|c|}{100} \\
\hline $\begin{array}{l}\text { Total suspended solids } \\
{\left[\mathrm{mg} \cdot \mathrm{dm}^{-3}\right]}\end{array}$ & $25^{2}$ & $30^{2}$ & $35^{2}$ & \multicolumn{3}{|c|}{100} & \multicolumn{2}{|r|}{100} & \multicolumn{3}{|c|}{100} & \multicolumn{3}{|c|}{100} & \multicolumn{3}{|c|}{100} \\
\hline Dissolved oxygen $\left[\mathrm{mg} \mathrm{O}_{2} \cdot \mathrm{dm}^{-3}\right]$ & $>70^{2}$ & $>50^{2}$ & $>30^{2}$ & \multicolumn{3}{|c|}{100} & \multicolumn{2}{|c|}{100} & \multicolumn{3}{|c|}{100} & \multicolumn{3}{|c|}{100} & \multicolumn{3}{|c|}{100} \\
\hline $\mathrm{BOD}_{5}\left[\mathrm{mg} \mathrm{O}_{2} \cdot \mathrm{dm}^{-3}\right]$ & $<3^{2}$ & $<5^{2}$ & $<7^{2}$ & \multicolumn{2}{|r|}{100} & & \multicolumn{2}{|r|}{100} & & 100 & & 71 & 71 & 86 & 57 & 86 & 100 \\
\hline COD-Mn $\left[\mathrm{mg} \mathrm{O}_{2} \cdot \mathrm{dm}^{-3}\right]$ & $25^{2}$ & $30^{2}$ & $30^{2}$ & \multicolumn{2}{|r|}{100} & & \multicolumn{2}{|c|}{100} & \multicolumn{3}{|c|}{100} & & 100 & & & 100 & \\
\hline $\begin{array}{l}\text { Electrolytic conductivity } \\
{\left[\mu \mathrm{S} \cdot \mathrm{cm}^{-1}\right]}\end{array}$ & $1000^{2}$ & $1000^{2}$ & $1000^{2}$ & & 100 & & & 100 & & 100 & & & 100 & & & 100 & \\
\hline $\mathrm{SO}_{4}^{2-}\left[\mathrm{mg} \cdot \mathrm{dm}^{-3}\right]$ & $250^{1}$ & $250^{1}$ & $250^{1}$ & & 100 & & & 100 & & 100 & & & 100 & & & 100 & \\
\hline $\mathrm{Cl}^{-}\left[\mathrm{mg} \cdot \mathrm{dm}^{-3}\right]$ & $250^{2}$ & $250^{2}$ & $250^{2}$ & & 100 & & & 100 & & 100 & & & 100 & & & 100 & \\
\hline Reaction $(\mathrm{pH})$ & $6.5-8.5^{2}$ & $\begin{array}{l}5.5- \\
9.0^{2}\end{array}$ & $\begin{array}{l}5.5- \\
9.0^{2}\end{array}$ & & 100 & & & 100 & 86 & & 100 & & 100 & & & 100 & \\
\hline $\mathrm{PO}_{4}^{3-}\left[\mathrm{mg} \cdot \mathrm{dm}^{-3}\right]$ & $0.4^{2}$ & $0.7^{2}$ & $0.7^{2}$ & & 100 & & & 100 & & 100 & & 86 & & 00 & & 100 & \\
\hline $\mathrm{NH}_{4}{ }^{+}\left[\mathrm{mg} \cdot \mathrm{dm}^{-3}\right]$ & $0.5^{2}$ & $1.5^{1}$ & $2.0^{1}$ & & 100 & & 86 & 100 & 57 & & 100 & 71 & 86 & 100 & 71 & 10 & 00 \\
\hline $\mathrm{NO}_{3}-\left[\mathrm{mg} \cdot \mathrm{dm}^{-3}\right]$ & $50^{1}$ & $50^{1}$ & $50^{1}$ & & 100 & & & 100 & & 100 & & & 100 & & & 100 & \\
\hline $\mathrm{Fe}\left[\mathrm{mg} \cdot \mathrm{dm}^{-3}\right]$ & $0.3^{1}$ & $2^{1}$ & $2^{2}$ & 57 & 100 & & 57 & 100 & 71 & & 100 & 57 & 71 & 71 & 86 & & 00 \\
\hline $\mathrm{Mn}\left[\mathrm{mg} \cdot \mathrm{dm}^{-3}\right]$ & $0.05^{2}$ & $0.1^{2}$ & $1^{2}$ & 43 & \begin{tabular}{|l|l|}
57 & 10
\end{tabular} & 005 & \begin{tabular}{l|l}
57 & 5
\end{tabular} & \begin{tabular}{l|l}
57 & 100
\end{tabular} & 43 & 71 & 100 & 43 & 57 & 100 & 71 & 86 & 100 \\
\hline
\end{tabular}

${ }^{1}$ for $95 \%$ samples, ${ }^{2}$ for $90 \%$ samples, red type indicates that the index value does not meet the requirements for a given water treatment category. 
Table 3. Usability of stream water as a natural environment for fish

\begin{tabular}{|c|c|c|c|c|c|c|c|c|c|c|c|c|}
\hline \multirow{4}{*}{ Indices } & \multirow{3}{*}{\multicolumn{2}{|c|}{$\begin{array}{c}\text { Values required for inland } \\
\text { waters as environment } \\
\text { for fish [Rozporządzenie } \\
\text { MŚ 2002a] }\end{array}$}} & \multicolumn{10}{|c|}{$\begin{array}{l}\text { Frequency of index values (\% of samples) in normative range } \\
\text { for a given fish category }\end{array}$} \\
\hline & & & \multicolumn{10}{|c|}{ Measurement-control point } \\
\hline & & & \multicolumn{2}{|l|}{1} & \multicolumn{2}{|l|}{2} & \multicolumn{2}{|l|}{3} & \multicolumn{2}{|l|}{4} & \multicolumn{2}{|l|}{5} \\
\hline & Salmonidae & Cyprinidae & salmon & carp & salmon & carp & salmon & carp & salmon & carp & salmon & carp \\
\hline Temperature $\left[{ }^{\circ} \mathrm{C}\right]$ & $21.5^{2}$ & $28.0^{2}$ & 100 & 100 & 100 & 100 & 100 & 100 & 100 & 100 & 100 & 100 \\
\hline Total suspended solids $\left[\mathrm{mg} \cdot \mathrm{dm}^{-3}\right]$ & \multicolumn{2}{|c|}{ average annual value $\leq 25$} & \multicolumn{2}{|c|}{$7.7^{3}$} & \multicolumn{2}{|c|}{$8.0^{3}$} & \multicolumn{2}{|c|}{$5.1^{3}$} & \multicolumn{2}{|c|}{$4.5^{3}$} & \multicolumn{2}{|c|}{$4.4^{3}$} \\
\hline \multirow{2}{*}{ Dissolved oxygen $\left[\mathrm{mg} \mathrm{O}_{2} \cdot \mathrm{dm}^{-3}\right]$} & $50 \% \geq 9$ & $50 \% \geq 8$ & 86 & 100 & 71 & 100 & 86 & 100 & 71 & 86 & 71 & 86 \\
\hline & $100 \% \geq 7$ & $100 \% \geq 5$ & 100 & 100 & 100 & 100 & 100 & 100 & 100 & 100 & 100 & 100 \\
\hline $\mathrm{BOD}_{5}\left[\mathrm{mg} \mathrm{O}_{2} \cdot \mathrm{dm}^{-3}\right]$ & $\leq 3^{1}$ & $\leq 6^{1}$ & 100 & 100 & 71 & 100 & 100 & 100 & 71 & 71 & 57 & 86 \\
\hline $\mathrm{pH}$ & \multicolumn{2}{|c|}{$6-9^{1}$} & \multicolumn{2}{|c|}{100} & \multicolumn{2}{|c|}{100} & \multicolumn{2}{|c|}{100} & \multicolumn{2}{|c|}{100} & \multicolumn{2}{|c|}{100} \\
\hline $\mathrm{N}-\mathrm{NH}_{4}{ }_{4}^{+}\left[\mathrm{mg} \cdot \mathrm{dm}^{-3}\right]$ & \multicolumn{2}{|c|}{$\leq 0.78^{1}$} & \multicolumn{2}{|c|}{100} & \multicolumn{2}{|c|}{100} & \multicolumn{2}{|c|}{100} & \multicolumn{2}{|l|}{71} & \multicolumn{2}{|l|}{71} \\
\hline $\mathrm{NO}_{2}^{-}\left[\mathrm{mg} \cdot \mathrm{dm}^{-3}\right]$ & $\leq 0.01^{1}$ & $\leq 0.03^{1}$ & 43 & 86 & 57 & 86 & 29 & 100 & 0 & 43 & 0 & 57 \\
\hline
\end{tabular}

${ }^{1}$ for $95 \%$ of samples, ${ }^{2}$ for $98 \%$ of samples, ${ }^{3}$ average value, red type indicates that the index value does not meet the requirements

Table 4. Pollution coefficients $\left(\mathrm{W}_{\mathrm{z}}\right)$ and pollution degree of stream water as determined on basis chosen indices of water quality

\begin{tabular}{|c|c|c|c|c|c|c|}
\hline \multirow{3}{*}{ Indices } & \multicolumn{5}{|c|}{$S D_{t}: S W_{t}, S W_{n-2}: S D_{n-2}$} & \multirow{3}{*}{\begin{tabular}{|c|} 
Norm \\
$\mathrm{SD}_{\mathrm{t}} \cdot \mathrm{SD}_{\mathrm{n}-2}$
\end{tabular}} \\
\hline & \multicolumn{5}{|c|}{ Measurement-control point } & \\
\hline & 1 & 2 & 3 & 4 & 5 & \\
\hline Temperature $\left[{ }^{\circ} \mathrm{C}\right]$ & 0.55 & 0.54 & 0.55 & 0.57 & 0.56 & 22 \\
\hline Total suspended solids $\left[\mathrm{mg} \cdot \mathrm{dm}^{-3}\right]$ & 0.31 & 0.32 & 0.20 & 0.18 & 0.18 & 25 \\
\hline Dissolved oxygen $\left[\mathrm{mg} \mathrm{O}_{2} \cdot \mathrm{dm}^{-3}\right]$ & 0.69 & 0.72 & 0.75 & 0.74 & 0.71 & 7 \\
\hline $\mathrm{BOD}_{5}\left[\mathrm{mg} \mathrm{O}_{2} \cdot \mathrm{dm}^{-3}\right]$ & 0.47 & 0.60 & 0.53 & 1.27 & 1.07 & 3 \\
\hline COD-Mn $\left[\mathrm{mg} \mathrm{O}_{2} \cdot \mathrm{dm}^{-3}\right]$ & 0.88 & 0.78 & 0.77 & 0.97 & 0.88 & 6 \\
\hline Electrolytic conductivity $\left[\mu \mathrm{S} \cdot \mathrm{cm}^{-1}\right]$ & 0.24 & 0.24 & 0.25 & 0.29 & 0.29 & 1000 \\
\hline Total dissolved solids $\left[\mathrm{mg} \cdot \mathrm{dm}^{-3}\right]$ & 0.37 & 0.35 & 0.38 & 0.47 & 0.45 & 500 \\
\hline $\mathrm{SO}_{4}{ }^{2-}\left[\mathrm{mg} \cdot \mathrm{dm}^{-3}\right]$ & 0.14 & 0.13 & 0.13 & 0.13 & 0.13 & 150 \\
\hline $\mathrm{Cl}^{-}\left[\mathrm{mg} \cdot \mathrm{dm}^{-3}\right]$ & 0.07 & 0.07 & 0.08 & 0.09 & 0.08 & 200 \\
\hline $\mathrm{Ca}^{2+}\left[\mathrm{mg} \cdot \mathrm{dm}^{-3}\right]$ & 0.33 & 0.34 & 0.38 & 0.39 & 0.32 & 100 \\
\hline $\mathrm{Mg}^{2+}\left[\mathrm{mg} \cdot \mathrm{dm}^{-3}\right]$ & 0.15 & 0.14 & 0.15 & 0.17 & 0.17 & 50 \\
\hline $\mathrm{PO}_{4}{ }^{3-}\left[\mathrm{mg} \cdot \mathrm{dm}^{-3}\right]$ & 0.40 & 0.50 & 0.45 & 1.25 & 0.50 & 0.2 \\
\hline $\mathrm{N}-\mathrm{NH}_{4}{ }_{4}^{+}\left[\mathrm{mg} \cdot \mathrm{dm}^{-3}\right]$ & 0.09 & 0.18 & 0.32 & 0.47 & 0.37 & 0.78 \\
\hline $\mathrm{N}-\mathrm{NO}_{3}^{-}\left[\mathrm{mg} \cdot \mathrm{dm}^{-3}\right]$ & 0.32 & 0.36 & 0.41 & 0.55 & 0.50 & 2.2 \\
\hline $\mathbf{w}_{\mathbf{z}}$ & 0.36 & 0.38 & 0.38 & 0.54 & 0.44 & - \\
\hline Pollution degree & clear & clear & clear & clear & clear & - \\
\hline
\end{tabular}

$\mathrm{W}_{\mathrm{z}}$ - water pollution coefficient calculated from the formula of Burchard and Dubaniewicz [1981],

$\mathrm{SW}_{\mathrm{t}}, \mathrm{SW}_{\mathrm{n}-2}, \mathrm{SD}_{\mathrm{t}}, \mathrm{SD}_{\mathrm{n}-2}-$ see formula (1)

strong relationship occurs between electrolytic conductivity and concentrations of magnesium, sodium, total suspended solids, iron, dissolved solids and potassium (Table 5). In case of these correlations, it may be observed that with increasing concentrations of total suspended solids and iron, electrolytic conductivity decreases, whereas with an increase in their water quality indices, water electrolytic conductivity grows (Figure 2).

\section{CONCLUSIONS}

1. On the basis of physicochemical indices supporting biological elements, the state of the Łososina river above Tymbark city was determined as very good, whereas it deteriorated below the city where its water was classified as good state due to $\mathrm{BOD}_{5}$ and phosphates concentrations. 
Table 5. Parameters of electrolytic conductivity (EC) correlation with concentration of some quality indices of analyzed river water

\begin{tabular}{|c|c|c|c|c|c|c|}
\hline Indices & $\mathrm{r}_{\mathrm{xy}}$ & $\mathrm{r}^{2}$ & $\mathrm{t}$ & $\mathrm{p}$ & $\mathrm{a}$ & $\mathrm{b}$ \\
\hline Total suspended solid (TSS) & -0.63 & $\mathbf{0 . 4 0}$ & -4.55 & $<0.001$ & -4.47 & 280.7 \\
\hline Total dissolved solids (TDS) & $\mathbf{0 . 5 6}$ & $\mathbf{0 . 3 1}$ & $\mathbf{3 . 7 2}$ & $<0.001$ & $\mathbf{0 . 7 8}$ & 102.8 \\
\hline $\mathrm{SO}_{4}^{2-}$ & -0.27 & 0.07 & -1.55 & 0.13 & -2.58 & 302.7 \\
\hline $\mathrm{Ca}^{2+}$ & -0.22 & 0.05 & -1.24 & 0.21 & -0.77 & 279.6 \\
\hline $\mathrm{Mg}^{2+}$ & 0.78 & 0.61 & 6.97 & $<0.001$ & 24.8 & 67.1 \\
\hline $\mathrm{Cl}^{-}$ & 0.12 & 0.01 & 0.67 & 0.51 & 1.96 & 224.4 \\
\hline $\mathrm{K}^{+}$ & 0.48 & 0.23 & 3.01 & 0.005 & 34.1 & 132.5 \\
\hline $\mathrm{Na}^{+}$ & 0.65 & 0.42 & 4.75 & $<0.001$ & 5.18 & 192.7 \\
\hline $\mathrm{Fe}$ & -0.58 & 0.33 & -3.93 & $<0.001$ & -33.6 & 273.2 \\
\hline $\mathrm{Mn}^{2+}$ & -0.09 & 0.007 & -0.48 & 0.64 & -22.4 & 256.5 \\
\hline
\end{tabular}

$r_{x y}$ - Pearson correlation coefficient, $r^{2}$ - coefficient of determination, $t$ - value Student's statistic, $p$ - probability level, a - regression coefficient, $\mathrm{b}$ - free word, red indicates that the relationship is statistically significant
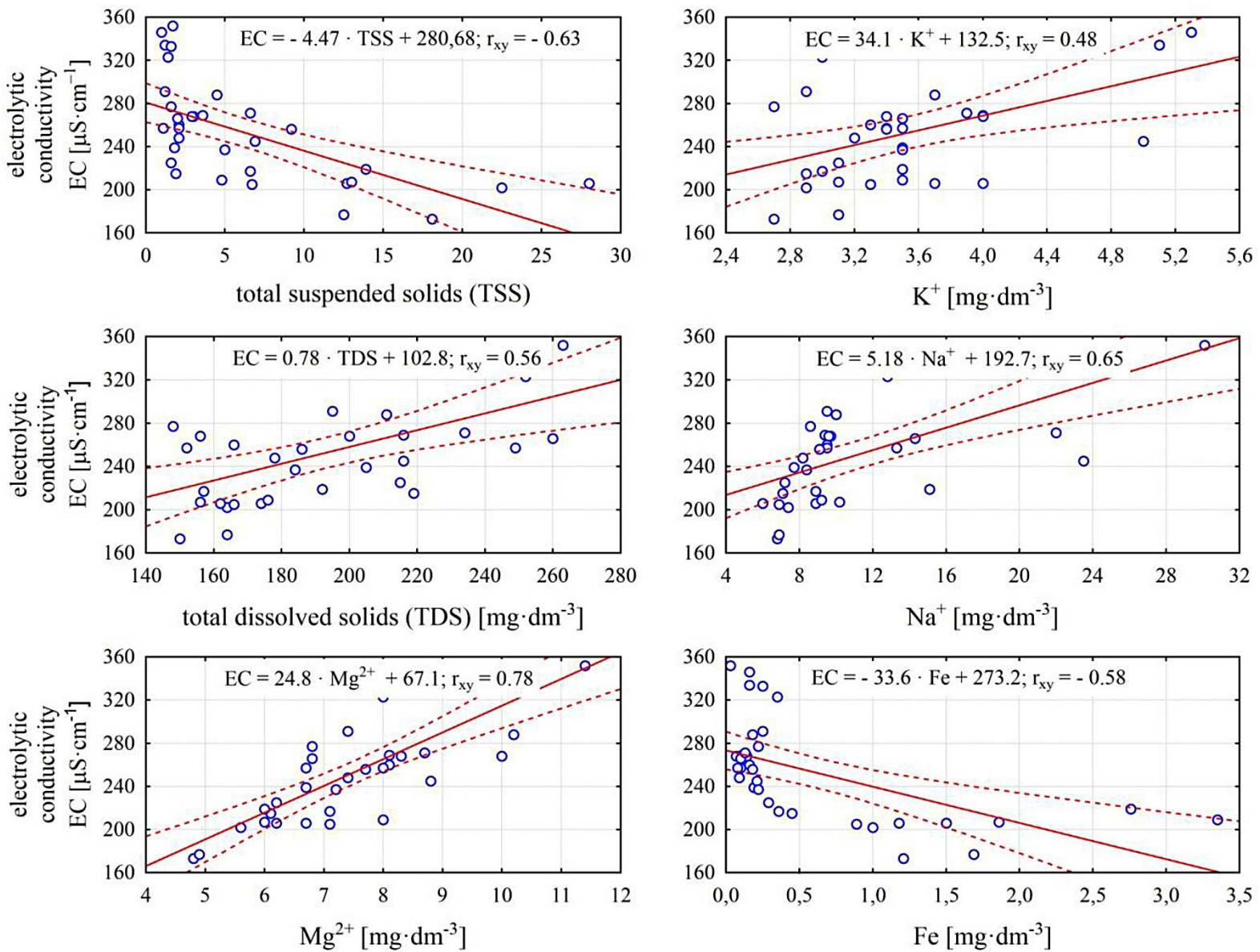

Figure 2. Dependence of electrolytic conductivity on the values of water quality indices

2. The Łososina river water above Tymbark city may be used for potable water supply to people, however, due to high manganese concentrations it must be subjected to an appropriate physical and chemical treatment. On the other hand, water analysed below the city (in points 4 and 5) did not meet the Ministry standards for A3 category of treatment for water supply to people.
3. Water on the investigated river stretch does not meet the requirements for inland waters, providing the natural habitat for salmonid and cyprinid fish because of exceeded nitrite concentrations. Only in point 3 the water may provide a natural habitat for cyprinid fish.

4. Correlation analysis revealed that electrolytic conductivity of the Łososina river water in- 
creases with increasing concentrations of total dissolved solids, magnesium, potassium and sodium but it decreases when the total suspended solids and iron concentrations increase.

\section{REFERENCES}

1. Ahiablame L. M., Engel B. A., Chaubey I. 2012. Effectiveness of Low Impact Development Practices: Literature Review and Suggestions for Future Research. Water Air Soil Pollut., 223, 42534273. DOI 10.1007/s11270-012-1189-2

2. Albrecht J. 2013. The Europeanization of water law by the Water Framework Directive: A secondo Chance for water planing In Germany. Land Use Policy. 30, 381-391.

3. Bogdał A., Kanownik W., Wiśnios M. 2012. Zmiany wartości i stężeń fizykochemicznych wskaźników jakościowych wód rzeki Prądnik-Białucha (Wyżyna Krakowsko-Częstochowska). Gaz, Woda i Technika Sanitarna, 8, 358-361.

4. Bogdał A., Policht-Latawiec A., Kołdras S. 2015. Zmiany wartości wskaźników jakości wody wraz z głębokością przy ujęciu wody pitnej ze zbiornika Dobczyce. Annual Set The Environment Protection (Rocznik Ochrony Środowiska), 17(2), 1239-1258.

5. Bourne A., Armstrong N., Jones G. 2002. A preliminary estimate of total nitrogen and total phosphorus loading to streams in Manitoba, Canada. Manitoba Conservation Report No 2002-04. Monitoba. Water Quality Management Section, 49.

6. Buda A., Jarynowski A. 2010. Life-time of correlations and its applications. 1, 5-21,

7. Burchard J., Dubaniewicz H. 1981. Współczynnik zanieczyszczenia jako miara kompleksowej oceny stopnia zanieczyszczenia wód powierzchniowych na przykładzie Pilicy i Bzury. Mat. IX Ogólnopolskiej Konf. Hydrograficznej, Łódź.

8. Chomutowska H., Wilamowski K. 2014. Analiza czystości wód rzeki Łutownia na terenie Puszczy Białowieskiej. Inżynieria Ekologiczna, 38, 117-128.

9. Czaban S. 2008. Klasyfikacja jakości wód powierzchniowych w Polsce. Annual Set The Environment Protection (Rocznik Ochrona Środowiska), 9, 259-269.

10. Derwich E., Benaabidate I., Zian A., Sadki O., Belghity D. 2010. Physicochemical characterization of waters of the alluvial layer of high Sebou downstream to its confluence with Oued fes. Larhyss J., 101-112.

11. EEA 2010. The European environment - state and outlook 2010. Copenhagen. 8-30.

12. El-Guamri Y., Belghyti D. 2006. Study of the physicochemical quality of raw waste water of the urban district of Saknia, rejected in the lake Fourat (Kénitra, Morocco). African J. Sci. and Environ., 1, 53-60.

13. Erle, E., Robert, P. 2010. Land-use and land-cover change. In: Encyclopedia of Earth Cutler J. Cleveland: Environmental Information Coalition, National Council for Science and the Environment. Washington DC.

14. Global Land Project (GLP) 2005. Science Plan and Implementation Strategy, IGBP Secretariat, Stockholm.

15. Grochowska J., Tandyrak R. 2007. Nitrogen and phosphorus compounds in Lake Pluszne. Arch. Environ. Protect., 33(1), 59-66.

16. ISO 5667-6:1997. Water quality. Sampling. Part 6: Guidance on sampling of rivers and Streams.

17. Kanownik W., Kowalik T., Bogdał A., Ostrowski K., Rajda W. 2011. Jakość i walory użytkowe wody potoku Szczyrzawy. Zesz. Probl. Postęp. Nauk Roln., 561, 65-79.

18. Kanownik W., Kowalik T., Bogdał A., Ostrowski K. 2013. Quality categories of stream water included in a Small Retention Program. Pol. J. Environ. Stud., 22(1), 159-165.

19. Kanownik W., Policht-Latawiec A. 2015. Changeability of oxygen and biogenic indices in waters flowing through the areas under various anthropopressure. Pol. J. Environ. Stud., 24(4), 1633-1640.

20. Kiryluk A., Rauba M. 2011. Wpływ rolnictwa na stężenie fosforu ogólnego w wodach powierzchniowych zlewni rzeki Śliny. Inżynieria Ekologiczna, 26, 122-132.

21. Kondracki J. 2013. Geografia regionalna Polski. Wyd. Nauk. PWN Warszawa.

22. Kruk A. 2007. Role of habitat degradation in determining fish distribution and abundance along the lowland Warta River, Poland. Journal of Applied Ichthyology, 23, 9-18.

23. Kuźniar A., Twardy S., Kowalczyk A. 2009. Changes in the pollution concentrations in the surface water of the Upper San against a background of land management. Polish Journal of Environmental Studies, 18(3a), 207-213.

24. Mladenović-Ranisavljevića I., Takića L., Vukovićb M., Nikolićb Đ., Živkovićc N., Milosavljevićd P. 2012. Multi-criteria ranking of the Danube water quality on its course through Serbia", Serbian Journal of Management, 7(2), 299-307.

25. Ostrowski K. 2010. Kształtowanie i ochrona zasobów wodnych w Polsce w nawiązaniu do Ramowej Dyrektywy Wodnej Unii Europejskiej. Zesz. Prob. Post. Nauk Rol, 548, 8-10.

26. Paczyński B., Sadurski A. 2007. Hydrogeologia Regionalna Polski. Tom I. Państwowy Instytut Geologiczny, Warszawa, 537. 
27. Policht-Latawiec A. 2012. Effect of treated sewage on water quality In the receiving Walters. Acta Hortic. Regiotec., 15, 46-49.

28. Policht-Latawiec A., Bogdał A., Kanownik W., Kowalik T., Ostrowski K., Gryboś P. 2014. Jakość i walory użytkowe wody małej rzeki fliszowej. Rocznik Ochrona Środowiska, 16(1), 546-561.

29. Policht-Latawiec A., Bogdał A., Kanownik W., Kowalik T., Ostrowski K. 2015. Variability of physicochemical properties of water of the transbboudary Poprad river. Journal of Ecological Engineering, 16(1), 100-109.

30. Policht-Latawiec A., Kanownik W. 2013. Jakość i walory użytkowe wody rzeki Szabasówki. Acta Sci. Pol., Formatio Circumiectus, 12(2), 93-102.

31. Raczyńska M., Machula S., Grzeszczyk-Kowalska A. 2013. Stan ekologiczny rzeki Stepnicy (Pomorze Zachodnie). Inżynieria Ekologiczna, 35, 46-59.

32. Radu V. M., Diacu E., Varduca A. 2013. Development of Procedures for Hydrochemical Monitoring Data Integration for the Danube River related to the Hydrological Conditions. Rev. Chim. (Bucharest), 64(3), 242-245.

33. Radu V.M., Diacu E., Ionescu P. 2014. Characterization of the eutrophication potential for the lower part of the Danube river. U.P.B. Sci. Bull., Series B, 76(4), 137-146.

34. Rozporządzenie Ministra Środowiska z dnia 4 października 2002 roku w sprawie wymagań, jakim powinny odpowiadać wody śródlądowe będące środowiskiem życia ryb w warunkach naturalnych (Dz. U. $2002 \mathrm{Nr}$ 176, poz. 1455), 2002a.

35. Rozporządzenie Ministra Środowiska z dnia 27 listopada 2002 r. w sprawie wymagań, jakim powinny odpowiadać wody powierzchniowe wykorzystywane do zaopatrzenia ludności w wodę przeznaczoną do spożycia (Dz. U. 2002 Nr 204, poz. 1728), 2002 b.

36. Rozporządzenie Ministra Środowiska z dnia 21 listopada 2013 r. zmieniające rozporządzenie MŚ z dnia 15 list. $2011 \mathrm{r}$. w sprawie form i sposobu prowadzenia monitoringu jednolitych części wód powierzchniowych i podziemnych (Dz. U. 2013 poz. 1558).

37. Rozporządzenie Ministra Środowiska z dnia 22 października 2014 r. w sprawie sposobu klasyfikacji stanu jednolitych części wód powierzchniowych oraz środowiskowych norm jakości dla substancji priorytetowych (Dz.U. 2014 poz. 1482).

38. Schoonover J. E., Lockaby B. 2006. Land cover impacts on stream nutrients and fecal coli form in the Lower Piedmont of West Georgia. J. Hydrol., 331, 371-382.

39. Sojka M., Murat-Błażejewska S. 2009. Stan fizykochemiczny i hydromorfologiczny małej rzeki nizinnej. Annual Set The Environment Protection (Rocznik Ochrona Środowiska), 11, 727-737.

40. Zampella R.A., Procopio N.A. 2009. Landscape Patterns and Water-Quality Relationships in New Jersey Pinelands Streams. Pinelands Commission, New Jersey, USA.

41. Zhang Y., Wu Y., Yu H., Dong Z., Zhang B. 2013. Trade-offs in designing water pollution trading policy with multiple objectives: A case study in the Tai Lake Basin, China. Environmental Science \& Policy, 33, 295-307.

42. Zieliński P., Górniak A., Bralski M. 2012. Wykorzystanie cech hydromorfologicznych do oceny stanu ekologicznego rzeki miejskiej. Inżynieria Ekologiczna, 29, 246-256. 